\title{
Some Classes of Functions with Exponential Decay in the Unit Ball of $\boldsymbol{C}^{n}$
}

By

\author{
OUYANG Caiheng*
}

\section{§1. Introduction}

One of the characterizing properties of the functions of bounded mean oscillation (BMO) is that their distribution functions have an exponential decay effect, i. e. the famous John-Nirenberg's theorem ${ }^{[1]}$. In 1980, Baernstein ${ }^{[2]}$ proved that the distribution functions of the non-tangential maximal functions decrease exponentially for a bounded subset of the Nevanlinna class in the unit disk, and as corollaries, he obtained an analytic form of John-Nirenberg's theorem with a weaker integrability assumption and pointed out that in the analytic category $\mathrm{BMO}$ is equivalent to $\mathrm{BMO}$ of logarithmic type. Long Ruilin and Yang $\mathrm{Le}^{[3]}$ obtained similar results to Baernstein's theorem in the $n$-dimensional real and complex ball by showing that $\mathrm{BMO}_{(\log )^{k}}=$ BMO for spaces of homogeneous type.

In this paper, we try to generalize a series of the famous Baernstein's results for the unit disk to the unit ball with respect to different topological structures applying Rudin's function theory in the unit ball of $C^{n[4]}$. In order to lead to the discussion, we define a class of point sets in the ball in $\S 2$, where we point out that there is a useful geometric property of the intersections of this class of sets and the admissible domains $D_{\alpha}(\zeta)$ defined by Korányi ${ }^{[5]}$. The key part of this paper will be found in $\S 3$, where the decay characterizations will be studied for a bounded subset of a function space larger than the Nevanlinna class, which shall be referred to as $H_{\varphi}$ class in the present article. Hence the maximal function in the admissible domain

Communicated by K. Saito, July 8, 1988.

* Institute of Mathematical Sciences, Academia Sinica, Wuhan 430071, PR of China. 
plays an important role in the proof. As the corollaries of the main results in $\S 3$, in $\S 4$, for the BMOA functions in the ball, we will prove the John-Nirenberg theorem with respect to the harmonic measure as well as the results derived thereof.

The main results had been reported in [6].

\section{§2. A Class of Point Sets in the Unit Ball}

Let $B$ be the unit ball $\left\{z \in \mathbb{C}^{n}:\langle z, z\rangle=\sum_{j=1}^{n} z_{j} \bar{z}_{j}<1\right\}$ and let $S$ be its boundary, i.e. the unit sphere. By Rudin's expression, the group $\mathscr{M}$ of the Möbius transformations of the unit ball is written as

$$
\mathscr{M}=\left\{\psi=\varphi_{a} U: \varphi_{a}=\frac{a-P_{a} z-s_{a} Q_{a} z}{1-\langle z, a\rangle}\right\},
$$

where $a \in B$, and $U$ are unitary transformations,

$$
P_{a} z=\left\{\begin{array}{ll}
\frac{\langle z, a\rangle}{\langle a, a\rangle} & a, a \neq 0, \\
0, & a=0,
\end{array} Q_{a}=I-P_{a}, \quad s_{a}=\left(1-|a|^{2}\right)^{1 / 2}\right.
$$

Obviously $\psi(0)=a$. Since, $\varphi_{a}$ maps the unit sphere $S$ into itself, $\mathscr{M}$ is also the transformation group of $S$ into itself. Note that $\psi \in \mathscr{M} \Leftrightarrow$ $\exists a, U \in \mathscr{U}$ (unitary group), such that $\phi(z)=\varphi_{a} U(z)$.

Now we define a class of point sets in the unit ball.

Definition. For $\beta>0$ and $a \in B(a \neq 0), \zeta \in S$, define

$$
R_{\beta, a}(\zeta)=\{z \in \bar{B}: \quad|1-\langle z, a\rangle| \leq \beta|1-\langle a, \zeta\rangle|\} .
$$

The point sets $R_{\beta, a}(\zeta)$ possess the following properties:

1) When $\beta<\frac{1-|a|}{1+|a|}, R_{\beta, a}(\zeta)$ is the empty set.

As a matter of fact, $\forall z \in B, \zeta \in S$, from Schwarz inequality,

$$
|1-\langle z, a\rangle| \geq 1-|z| \cdot|a| \geq 1-|a|
$$

and

$$
\beta|1-\langle a, \zeta\rangle| \leq \beta(1+|\langle a, \zeta\rangle|) \leq \beta(1+|a|) .
$$

Thus, when $1-|a|>\beta(1+|a|), \quad|1-\langle z, a\rangle|>\beta|1-\langle a, \zeta\rangle|$.

2) When $\beta \geq \frac{1+|a|}{1-|a|}$, for every fixed $\zeta \in S, R_{\beta, a}(\zeta)$ fills the closed ball $\bar{B}$. 
3) When $\beta \geq 1+|a|, a \in R_{\beta, a}(\zeta)$ for any $\zeta \in S$.

The reason of properties 2) and 3 ) is the same as 1).

4) For any unitary transformation, $U\left(R_{\beta, a}(\zeta)\right)=R_{\beta, U a}(U \zeta)$.

This is obvious from the defining equation of $R_{\beta, a}(\zeta)$. Hence, without loss of generality, take $\zeta=e_{1}=(1,0, \ldots, 0)$, and especially put $a=r e_{1}(0<r<1)$, then

$$
R_{\beta, r e_{1}}\left(e_{1}\right)=\left\{z \in \bar{B}:\left|1-r z_{1}\right| \leq \beta(1-r)\right\} .
$$

Again denote $z_{1}=\rho e^{i \theta}$. For instance, if we take $r=\frac{2}{3}, \beta=3$, then

$$
R_{3,(2 / 3) e_{1}}\left(e_{1}\right)=\left\{z \in \bar{B}: \quad \rho \leq 3 \cos \theta,-\frac{\pi}{2} \leq \theta \leq \frac{\pi}{2}\right\} .
$$

It is the point set in the closed unit ball where those first variables $z_{1}$ belong to the closed disk $\rho \leq 3 \cos \theta$. This special case provided some geometrically intuitional information for point sets $R_{\beta, a}(\zeta)$.

In the following, the relationship between $R_{\beta, a}(\zeta)$ and the admissible domain is discussed. According to the Rudin's ${ }^{[4]}$ expression

$$
D_{\alpha}(\zeta)=\left\{z \in B: \quad|1-\langle z, \zeta\rangle|<\frac{\alpha}{2}\left(1-|z|^{2}\right), \quad \zeta \in S, \alpha>1\right\} .
$$

In the discussion of function theory in the unit ball, its situation is similar to Stolz region $\Gamma_{\alpha}(\theta)$ in the unit disk. From the defining equation of $D_{\alpha}(\zeta)$, it is easy to know that for radial point $a=r_{1} \zeta(0$ $\left.<r_{1}<1\right)$ of $\zeta, a \in D_{\alpha}(\zeta)$, when $r_{1}>\frac{2}{\alpha}-1$, therefore, radial points $z=$ $r \zeta\left(r_{1} \leq r<1\right)$ belong to $D_{\alpha}(\zeta)$. Combining property 3$)$ of point set $R_{\beta, a}(\zeta)$, then, when $\alpha>\frac{2}{1+r_{1}}, \beta \geqq 1+r_{1}, z=r \zeta\left(r_{1} \leqq r<1\right)$ belongs to $D_{\alpha}$

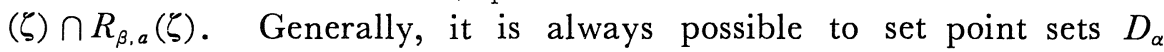
$(\zeta) \cap R_{\beta, a}(\zeta)$ to be nonempty when the values of $\alpha$ and $\beta$ are suitably chosen. Thus we have

Proposition. If $\phi \in \mathscr{M}$, choose $\alpha$ and $\beta$ so that $\alpha \beta>1$ and $D_{\alpha}(\zeta) \cap$ $R_{\beta, a}(\zeta)$ is nonempty, then

$$
D_{\alpha}(\zeta) \cap R_{\beta, a}(\zeta) \subset \psi\left(D_{\alpha \beta}(\xi)\right),
$$

where $\phi(\xi)=\zeta$.

Proof. It is pointed out in theorem 2.2.5 of [4] that if $\phi \in$ Aut 
$(B)$, and $a=\psi^{-1}(0)$, then there exists a unique $U$, such that $\psi=$ $U \varphi_{a} ;$ and

$$
1-\langle\psi(z), \psi(\zeta)\rangle=\frac{(1-\langle a, a\rangle)(1-\langle z, \zeta\rangle)}{(1-\langle z, a\rangle)(1-\langle a, \zeta\rangle)}, \text { for all } z, \zeta \in \bar{B} .
$$

Since, if $\zeta \in S, z \in D_{\alpha}(\zeta) \cap R_{\beta, a}(\zeta)$, then

$$
\begin{aligned}
& |1-\langle\psi(z), \quad \psi(\zeta)\rangle|\left\langle\frac{|1-\langle a, a\rangle| \cdot(\alpha / 2)\left(1-|z|^{2}\right)}{|1-\langle z, a\rangle| \cdot(1 / \beta)|1-\langle z, a\rangle|}\right. \\
= & \frac{\alpha \beta}{2} \cdot \frac{|1-\langle a, a\rangle| \cdot|1-\langle z, z\rangle|}{|1-\langle z, a\rangle|^{2}}=\frac{\alpha \beta}{2}\left(1-|\psi(z)|^{2}\right) .
\end{aligned}
$$

But then $\alpha \beta>1$, thus $\psi(z) \in D_{\alpha \beta}(\psi(\zeta))$. Then it is deduced that

$$
\psi\left(D_{\alpha}(\zeta) \cap R_{\beta, a}(\zeta)\right) \subset D_{\alpha \beta}(\psi(\zeta)) .
$$

Notice that $\varphi_{a}$ is the $1-1$ mapping on $\bar{B}$, then

$$
D_{\alpha}(\zeta) \cap R_{\beta, a}(\zeta) \subset \psi^{-1}\left(D_{\alpha \beta}(\xi)\right), \quad \xi=\psi(\zeta) .
$$

However, since $\varphi_{a}^{-1}=\varphi_{a}$, then $\psi^{-1}=\left(U \varphi_{a}\right)^{-1}=\varphi_{a} U^{-1} \in \mathscr{M}$, denoting $\phi$ as $\psi^{-1}$, then $\phi(\xi)=\zeta$. Thus

$$
D_{\alpha}(\zeta) \cap R_{\beta, a}(\zeta) \subset \psi\left(D_{\alpha \beta}(\xi)\right) .
$$

Remark. This result means that under Möbius transformation, the admissible domain for a point $\xi$ on the unit sphere includes the intersection of the admissible domain $D_{\alpha}(\zeta)$ and $R_{\beta, a}(\zeta)$ for its image point $\zeta$. This is an interesting fact for the complex geometry of $\boldsymbol{C}^{n}$. We are to use it below.

\section{§3. Baernstein Theorem for the $\boldsymbol{H}_{\varphi}$ Class of Functions}

Let $E$ be a measurable set on the sphere $S$, for $a \in B$, we define ${ }^{[6]}$

$$
\mu_{a}(E)=\int_{E} d \mu_{a}(\zeta)=\int_{E} \frac{\left(1-|a|^{2}\right)^{n}}{|1-\langle a, \zeta\rangle|^{2 n}} d \sigma(\zeta)
$$

to be the harmonic measure of $E$ at $a$ with respect to $B$. The integrand function at the right-handed side of the above equation is the Poisson integral kernel $P(a, \zeta)$ in $B$. For any $E \subset S$, the normalized Lebesgue measure $\sigma(E)=\mu_{0}(E)$.

As in [4], let $\varphi:[-\infty, \infty) \rightarrow[0, \infty)$ be a nondecreasing convex function, not identically 0 , the class of functions in $B$ 


$$
H_{\varphi}(B)=\left\{\begin{array}{l}
f \text { is holomorphic in } B \\
I_{1}(f)=\sup _{0<r<1} \int_{S} \varphi\left(\log \left|f_{r}\right|\right) d \sigma<\infty, f_{r}=f(r \zeta) .
\end{array}\right.
$$

If $\varphi(x)=x^{+}=\max (0, x)$, then $H_{\varphi}(B)=N(B)$. Here $N(B)$ denotes the Nevanlinna class

$$
N(B)=\left\{\begin{array}{l}
f \text { is holomorphic in } B \\
T(f)=\sup _{0<r<1} \int_{S} \log ^{+}\left|f_{r}\right| d \sigma<\infty .
\end{array}\right.
$$

If $\varphi(x)=e^{p_{x}}$, then $H_{\varphi}(B)=H^{p}(B), 0<p<\infty$. When $p_{1}>p_{2}$, we have $H^{p_{1}} \subset H^{p_{2}} \subset N$.

For the admissible domain $D_{\alpha}(\zeta)$ and a continuous function $F$ in $B$, the maximal function in the admissible domain is defined ${ }^{[4]}$ by

$$
\left(M_{\alpha} F\right)(\zeta)=\sup \left\{|F(z)|: z \in D_{\alpha}(\zeta)\right\} \text {. }
$$

Obviously, if there is a $K$-limit $F^{*}(\zeta)=\lim _{z \in D_{\alpha}(\zeta), z \rightarrow \zeta} F(z)$ of function $F$ for a point $\zeta$, then $\left(M_{\alpha} F\right)(\zeta) \geq\left|F^{*}(\zeta)\right|$.

Lemma 1. If $f \in H_{\varphi}$, then for every $t$ satisfying $\varphi(\log t)>0$, we have the following inequality

$$
\mu_{0}\left(\left\{\zeta \in S:\left(M_{\alpha} f\right)(\zeta)>t\right\}\right)<\frac{C(\alpha)}{\varphi(\log t)} I_{1}(f),
$$

where $C(\alpha)$ is a constant depending on $\alpha$.

Proof. If $f \in H_{\varphi}$, then $I_{1}(f)=\sup _{0<r<1} \int_{S} \varphi\left(\log \left|f_{r}\right|\right) d \sigma<\infty$. Hence from theorem 5.6.2. (a) in [4], there is a positive measure $\nu$ on $S$, such that $u=P[\nu]$ is the least $\mathscr{M}$-harmonic majorant of $\varphi(\log |f|)$, and $\|\nu\|=I_{1}(f)$, here $\|\nu\|=|\nu|(S)$ is the total variation measure of $\nu$ on $S$.

From the definition of the least $\mathscr{M}$-harmonic majorant function, for every $z \in B$, there is $\varphi(\log |f|) \leq P[\nu]$. However, since the compound function $\varphi \circ \log$ is nondecreasing, so that for definite $D_{\alpha}(\zeta)$ we have $\varphi\left(\log \left(M_{\alpha} f\right)(\zeta)\right) \leq\left(M_{\alpha} P[\nu]\right)(\zeta)$. Therefore, if $\left(M_{\alpha} f\right)(\zeta)>t$, then

$$
\left(M_{\alpha} P[\nu]\right)(\zeta) \geq \varphi(\log t)
$$

Hence

$$
\left\{\zeta \in S:\left(M_{\alpha} f\right)(\zeta)>t\right\} \subset\left\{\zeta \in S:\left(M_{\alpha} P[\nu]\right)(\zeta) \geq \varphi(\log t)\right\} .
$$


Thus it is only necessary to prove

$$
\mu_{0}\left(\left\{\zeta \in S:\left(M_{\alpha} P[\nu]\right)(\zeta) \geq \varphi(\log t)\right\}\right)<\frac{C(\alpha)}{\varphi(\log t)} I_{1}(f) .
$$

Now let us prove this inequality. According to Rudin [4, p68], define the maximal function of a complex measure on $S$ by

$$
(M \nu)(\zeta)=\sup _{\delta>0} \frac{|\nu| Q(\zeta, \delta)}{\sigma\left(Q_{\delta}\right)}
$$

where $Q_{\delta} \cong Q(\zeta, \delta)=\left\{\eta \in S:|1-\langle\zeta, \eta\rangle|^{1 / 2}<\delta, \zeta, \eta \in S, \delta>0\right\}$ is the "ball" on $S, \zeta$ is the center of the ball and $\delta$ is the radius. Then one has the corresponding inequality of weak type $(1,1)$ :

$$
\mu_{0}(\{\zeta \in S:(M \nu)(\zeta)>\tau\}) \leq A_{3} \tau^{-1}|| \nu \mid \text {, for every } \tau>0 .
$$

A result of Korányi [5] is expressed as

$$
\left(M_{\alpha} P[\nu]\right)(\zeta) \leq A(\alpha)(M \nu)(\zeta) .
$$

Combining this inequality and the above inequality of weak type $(1,1)$, suitably enlarging constant $A(\alpha)$, then

$$
\begin{aligned}
& \mu_{0}\left(\left\{\zeta \in S:\left(M_{\alpha} P[\nu]\right)(\zeta) \geq \varphi(\log t)\right\}\right) \\
& \leq \mu_{0}\left(\left\{\zeta \in S:(M \nu)(\zeta)>\frac{\varphi(\log t)}{A(\alpha)}\right\}\right) \\
& \leq \frac{A_{3} A(\alpha)}{\varphi(\log t)}\|\nu\|<\frac{C(\alpha)}{\varphi(\log t)} I_{1}(f) .
\end{aligned}
$$

Similar to that of [2], we introduce the set of functions

$$
\mathscr{M}(f)=\{g: g(z)=f \circ \psi(z)-f \circ \phi(0), \phi \in \mathscr{M}\} .
$$

Then we have

Theorem 1. Suppose that $f(z)$ is holomorphic in $B$, if $\mathscr{M}(f)$ is a bounded subset of $H_{\varphi}$, then for every $g(z) \in \mathscr{M}(f)$, we have

$$
\mu_{0}\left(\left\{\eta \in S:\left(M_{\alpha} g\right)(\eta)>t\right\}\right)<K e^{-\lambda t},
$$

where $K$ denotes the absolute constant, and $\lambda=C e^{-\varphi^{-1}\left(c(\alpha) \sigma_{\varphi}(f)\right)}, \sigma_{\varphi}(f)=$ $\sup \left\{I_{1}(g): g \in \mathscr{M}(f)\right\}$ for an increasing convex function.

Proof. Before beginning the proof, we ought to have some necessary preparations.

Let $G$ be any compact set on $S$ whose measure is non-zero, and 
employing finite many balls $\{Q\}$, with the centers $\zeta \in G$, cover $G$. Since $\{Q\}$ are only finitely many, suitably choosing the positions of the centers of those balls and the radii of those balls, it is always possible that $\sigma(J) \geqq \frac{1}{M} \sigma(Q)$ holds for every $Q$, where $J=G \cap Q, M$ is a larger positive constant given in advance. Next using the covering lemma ${ }^{[4]}$, we choose a disjoint subcollection $\Gamma=\left\{Q_{i}\right\}$ from $\{Q\}$, thus $\sigma(G) \leq A_{3} \sum_{\Gamma} \sigma\left(Q_{i}\right)$. Writing

$$
J_{i}=G \cap Q_{i} .
$$

Obviously $J_{i}$ are nonempty and pairwise disjoint, and each $J_{i}$ has the possibility to be composed of the countable many of pathconnected components. Denote $M_{1}$ as the product $A_{3} M$, then

$$
\sigma(G) \leq M_{1} \sum_{\Gamma} \sigma\left(J_{i}\right)
$$

In addition, for every ball $Q_{i}\left(\zeta_{i}, \delta_{i}\right)$ in $\Gamma$, we might as well suppose $\sigma\left(Q_{i}\right)<1 / 4$ and take $a_{i}=r_{i} \zeta_{i}, r_{i}=1-\delta_{i}^{2}$

When $\eta$ belongs to certain $Q_{i}$ in the above-mentioned text, as (2) in [7]

$$
d \mu_{a}(\eta) \geq C_{1} \frac{d \sigma(\eta)}{\sigma\left(Q_{i}\right)}
$$

where $G_{1}$ only depends on the dimension $n(>1)$. Thus for every $J_{i} \subset Q_{i}$, there is

$$
\mu_{0}\left(J_{i}\right) \leq C_{1}^{-1} \mu_{a}\left(J_{i}\right) \mu_{0}\left(Q_{i}\right) .
$$

When $\mathscr{M}(f)$ is bounded in $H_{\varphi}$ class, set $\sigma_{\varphi}(f)=\sup \left\{I_{1}(g): g \in\right.$ $\mathscr{M}(f)\}$. For constant $M_{1}$ in (1) and constant $C_{1}$ in (2), choose $\tau$ large enough so that

$$
\frac{C(\alpha) \sigma_{\varphi}(f)}{\varphi(\log \tau)} \leq \min \left(\frac{C_{1}}{2 M_{1}}, \frac{1}{4}\right)
$$

where $C(\alpha)$ is the constant in Lemma 1 .

Now the proof of the theorem is carried out successively.

$1^{\circ}$ For the fixed $g(z) \in \mathscr{M}(f)$, define

$$
E_{k}=\left\{\eta \in S:\left(M_{\alpha} g\right)(\eta)>k \tau\right\}, k=1,2, \ldots
$$

Obviously $E_{k+1} \subset E_{k}$. Assume that $E_{k+1}$ is nonempty, let $G$ be a compact subset of the open set $E_{k+1}$ whose measure is non-zero, same as the statement in the preparation in this proof, cover $G$ by those 
balls $\{Q\}$ which are included in $E_{k}$ with their centers $\zeta \in G$, and also write $\Gamma=\left\{Q_{i}\right\}$ to be the disjoint subcollection chosen by the covering lemma.

$2^{\circ}$ Now let us prove

$$
\mu_{0}\left(E_{k+1}\right) \leq(1 / 2) \mu_{0}\left(E_{k}\right), \quad k \geq 1 .
$$

Since $f(z)$ is holomorphic in $B$, and $\varphi_{a}: \bar{B} \rightarrow \bar{B}$ is the holomorphic mapping, thus $g(z)$ is holomorphic function in $B$. Hence in closed ball $\bar{B}_{r_{0}}=\left\{z \in B:|z| \leq r_{0}\right\}$ with its radius $r_{0}$ near to 1 (i. e. $1-r_{0}=$ $\mathrm{o}\left(\delta_{i}^{2}\right)$ ), for enough large $\tau$ in (3), we have $|g(z)| \leq k \tau$. Therefore when $\eta \in Q_{i}\left(\subset E_{k}\right)$,

$$
\begin{aligned}
\left(M_{\alpha} g\right)(\eta) & =\sup \left\{|g(z)|: z \in D_{\alpha}(\eta)\right\} \\
& =\sup \left\{|g(z)|: z \in D_{\alpha}(\eta) \cap \bar{B}_{r_{0}}^{c}\right\}
\end{aligned}
$$

where $\bar{B}_{r_{0}}^{c}=\left\{z \in B:|z|>r_{0}\right\}$.

For the given $\alpha$, when $z \in D_{\alpha}(\eta) \cap \bar{B}_{r_{0}}^{c}$, there is

$$
|1-\langle z, \eta\rangle|<\frac{\alpha}{2}\left(1-|z|^{2}\right)<\alpha(1-|z|)<\alpha\left(1-r_{0}\right)<\delta_{i}^{2},
$$

if not, then suitably increase the value of $r_{0}$. However

$$
\left|1-\left\langle a_{i}, \eta\right\rangle\right| \geq 1-\left|a_{i}\right| \cdot|\eta|=1-r_{i}=\delta_{i}^{2},
$$

hence

$$
\left|1-\left\langle z, a_{i}\right\rangle\right|^{1 / 2} \leq|1-\langle z, \eta\rangle|^{1 / 2}+\left|1-\left\langle a_{i}, \eta\right\rangle\right|^{1 / 2}\left\langle 2\left|1-\left\langle a_{i}, \eta\right\rangle\right|^{1 / 2} .\right.
$$

By the definition of set $R_{\beta, a}(\zeta)$, we know that $z \in R_{4, a_{i}}(\eta)$. Therefore, we deduce that $D_{\alpha}(\eta) \cap \bar{B}_{r_{0}}^{c} \subset R_{4, a_{i}}(\eta)$ which could be written as

$$
D_{\alpha}(\eta) \cap \bar{B}_{r_{0}}^{c} \subset D_{\alpha}(\eta) \cap R_{4, a_{i}}(\eta) .
$$

Defining $\psi_{a_{i}}=\varphi_{a_{i}} U \in \mathscr{M}$ and $g_{i}(z)=g \circ \psi_{a_{i}}(z)-g \circ \psi_{a_{i}}(0)=g \circ \psi_{a_{i}}(z)-g\left(a_{i}\right)$, and applying the proposition in $\S 2$, then we have the following inclusion relation

$$
D_{\alpha}(\eta) \cap \bar{B}_{r_{0}}^{c} \subset \psi_{a_{i}}\left(D_{4 \alpha}(\xi)\right),
$$

where $\phi_{a_{i}}(\xi)=\eta$.

For $J_{i}$ is nonempty, then, when $\eta \in J_{i}\left(\subset E_{k+1}\right)$, from Eq. (5) and (6)

$$
(k+1) \tau<\left(M_{\alpha} g\right)(\eta)=\sup \left\{|g(z)|: z \in D_{\alpha}(\eta) \cap \bar{B}_{r_{0}}^{c}\right\}
$$




$$
\begin{aligned}
& \leq \sup \left\{|g(z)|: z \in \phi_{a_{i}}\left(D_{4 \alpha}(\xi)\right)\right\} \\
& =\sup \left\{\left|g \circ \psi_{a_{i}}(z)\right|: z \in D_{4 \alpha}(\xi)\right\} .
\end{aligned}
$$

Since we have taken $a_{i}=r_{i} \zeta_{i}, r_{i}=1-\delta_{i}^{2} \ll r_{0}$, thus $a_{i} \in \bar{B}_{r_{0}}$, therefore $\left|g\left(a_{i}\right)\right| \leq k \tau$. Thus, there is

$$
\begin{aligned}
& \left(M_{4 \alpha} g_{i}\right)(\xi)=\sup \left\{\left|g_{i}(z)\right|: z \in D_{4 \alpha}(\xi)\right\} \\
= & \sup \left\{\left|g \circ \phi_{a_{i}}(z)-g\left(a_{i}\right)\right|: z \in D_{4 \alpha}(\xi)\right\} \\
\geq & \sup \left\{\left|g \circ \phi_{a_{i}}(z)\right|-\left|g\left(a_{i}\right)\right|: z \in D_{4 \alpha}(\xi)\right\} \\
> & (k+1) \tau-k \tau=\tau,
\end{aligned}
$$

noting that $\eta=\psi_{a_{i}}(\xi)$, this means

$$
J_{i} \subset \psi_{a_{i}}\left(\left\{\xi \in S:\left(M_{4 \alpha} g_{i}\right)(\xi)>\tau\right\}\right) .
$$

Since $g_{i} \in \mathscr{M}(f)$, applying Lemma 1 and (3), we obtain

$$
\mu_{0}\left(\left\{\xi \in S:\left(M_{4 \alpha} g_{i}\right)(\xi)>\tau\right\}\right) \leq \frac{C_{1}}{2 M_{1}} .
$$

Denote $I=\left\{\xi \in S:\left(M_{4 \alpha} g_{i}\right)(\xi)>\tau\right\}$, combining (7) and (8), and applying Eq. (8. 11) in [8], then

$$
\mu_{a_{i}}\left(J_{i}\right) \leq \mu_{a_{i}}\left(\phi_{a_{i}}(I)\right)=\mu_{\psi_{a_{i}}}(0)\left(\phi_{a_{i}}(I)\right)=\mu_{0}(I) \leq \frac{C_{1}}{2 M_{1}} .
$$

Together with (2) and (9), we deduce

$$
\mu_{0}\left(J_{i}\right) \leq C_{1}^{-1} \cdot \frac{C_{1}}{2 M_{1}} \mu_{0}\left(Q_{i}\right)=\frac{1}{2 M_{1}} \mu_{0}\left(Q_{i}\right) \text {. }
$$

Now let us discuss as above for every $J_{i}$, and permute the order of all the path-connected components of $J_{i}$, it does not matter to write it as $J_{i}$. Applying (10), then

$$
\mu_{0}(G) \leq M_{1} \sum_{\Gamma} \mu_{0}\left(J_{i}\right) \leq(1 / 2) \sum_{\Gamma} \mu_{0}\left(Q_{i}\right) \leq(1 / 2) \mu_{0}\left(E_{k}\right) .
$$

Now (4) follows by taking the supremum over all compact subsets $G \subset E_{k+1}$.

$3^{\circ}$ Lemma 1 and (3) are to be used once more, then there is $\mu_{0}\left(E_{1}\right)<1 / 4<1 / 2$. Thus it can be deduced from (4) inductively that

$$
\mu_{0}\left(E_{k}\right) \leq(1 / 2) \mu_{0}\left(E_{k-1}\right) \leq \ldots \leq \frac{1}{2^{k-1}} \mu_{0}\left(E_{1}\right) \leq \frac{1}{2^{k}} .
$$

For any $t>0$, when $t \geq \tau$, there always exists certain $k$, such that $k \tau \leq t<(k+1) \tau$, hence 


$$
\begin{aligned}
& \mu_{0}\left(\left\{\eta \in S:\left(M_{\alpha} g\right)(\eta)>t\right\}\right) \leq \mu_{0}\left(E_{k}\right) \\
\leq & 2^{-k}=2 e^{-\left(\frac{1}{\tau} \log 2\right) \cdot(k+1) \tau}<2 e^{-\left(\frac{1}{\tau} \log 2\right) t} ;
\end{aligned}
$$

again when $0<t<\tau$, we have also

$$
\begin{aligned}
& \mu_{0}\left(\left\{\eta \in S:\left(M_{\alpha} g\right)(\eta)>t\right\}\right) \leq \mu_{0}(S) \\
= & 1=2 e^{-\left(\frac{1}{\tau} \log 2\right) \tau}<2 e^{-\left(\frac{1}{\tau} \log 2\right) t} .
\end{aligned}
$$

Thus for any $t>0$, there is

$$
\mu_{0}\left(\left\{\eta \in S:\left(M_{\alpha} g\right)(\eta)>t\right\}\right)<K e^{-\lambda t},
$$

where $K=2$, for increasing convex $\varphi, \lambda=\frac{1}{\tau} \log 2=C e^{-\varphi^{-1}\left(C(\alpha) \sigma_{\varphi}(f)\right)}$. Now the theorem is proved.

Remark. For general nondecreasing convex functions, it does not seem that it is easy to yield the converse of Theorem 1 , however, for some $H_{\varphi}$ defined by $\varphi$, the converse is also true, just as the $N$ class to be proved in the following.

Before the further discussion is going on, let us first introduce the elementary Lemma used in [2]:

Lemma 2. Let $h$ be a nonnegative measurable function on some measure space $(\Omega, \mathscr{F}, \mu)$. The distribution function $\Lambda(t)$ of $h$ is defined as

$$
\Lambda(t)=\mu(\{x \in \Omega: h(x)>t\}), t>0 .
$$

Then the following two equalities hold

(i) $\int_{\Omega} h^{p} d \mu=-\int_{0}^{\infty} t^{p} d \Lambda(t)=p \int_{0}^{\infty} t^{p-1} \Lambda(t) d t, 0<p<\infty$.

(ii) $\int_{\Omega}\left(\log ^{+} \frac{h}{\rho}\right) d \mu=-\int_{\rho}^{\infty}\left(\log \frac{t}{\rho}\right) d \Lambda(t)=\int_{\rho}^{\infty} t^{-1} \Lambda(t) d t, 0<\rho<\infty$.

Similarly, we could obtain the following lemma by the standard argument of real function theory, i. e. the simple functions approximate to an arbitrary measurable function.

Lemma 3. $h$ and its distribution function $\Lambda(t)$ are defined as above and $\mu(\Omega)=1$, then 


$$
\int_{\Omega} e^{\rho h} d \mu=-\int_{0}^{\infty} e^{\rho t} d \Lambda(t) \leq 1+\rho \int_{0}^{\infty} e^{\rho t} \Lambda(t) d t, \quad 0<\rho<\infty
$$

As a deduction of Theorem 1 and Lemma 2, there is

Theorem 2. Suppose that $f(z)$ is holomorphic in $B$, then the following are equivalent:

(a) $\mathscr{M}(f)$ is bounded in $N$.

(b) there exists an absolute constant $K$ and a constant $\lambda=\lambda(\alpha, f)$ such that for every $g \in \mathscr{M}(f)$ and $t>0$

$$
\mu_{0}\left(\left\{\eta \in S:\left(M_{\alpha} g\right)(\eta)>t\right\}\right)<K e^{-\lambda t} .
$$

Proof. (a) $\Rightarrow(\mathrm{b})$ is the implication following directly from Theorem 1. In fact, if take $\varphi(x)=x^{+}=\max (0, x)$, then $H_{\varphi}(B)=N(B)$. Thus by Theorem 1, it is clear that $(\mathrm{a}) \Rightarrow(\mathrm{b})$. At this moment, $\lambda=$ $C e^{-C(\alpha) \sigma(f)}$, where $\sigma(f)=\sup \{T(g): g \in \mathscr{M}(f)\}$.

(b) $\Rightarrow$ (a). Take $\Omega=S$ in Lemma 2(ii), $\rho=1$, and set $h=M_{\alpha} g$, $\Lambda(t)=\mu_{0}\left(\left\{\eta \in S:\left(M_{\alpha} g\right)(\eta)>t\right\}\right)$. If (b) holds, then

$$
\int_{S}\left(\log ^{+} M_{\alpha} g\right) d \mu_{0}=\int_{1}^{\infty} t^{-1} \Lambda(t) d t<\int_{1}^{\infty} t^{-1} K e^{-\lambda t} d t<\infty .
$$

For the sake of definitivity, firstly, let $\alpha \geq 2$, when $0<r<1$ there is $r \eta \in D_{\alpha}(\eta)$. Thus for every $\eta \in S,\left|g_{r}(\eta)\right| \leq\left(M_{\alpha} g\right)(\eta)$. Therefore, for every $g \in \mathscr{M}(f)$,

$$
\sup _{0<r<1} \int_{S}\left(\log ^{+}\left|g_{r}\right|\right) d \sigma \leq \int_{S}\left(\log ^{+} M_{\alpha} g\right) d \mu_{0}<\infty
$$

that is, $\mathscr{M}(f)$ is bounded in $N$.

When $1<\alpha<2$, take $r_{1}=\frac{2}{\alpha}-1$, clearly $0<r_{1}<1$. Since the holomorphic function $g_{r}$ is bounded in the closed ball $\bar{B}_{r_{1}}$, hence, when $r \leq r_{1}$, it is also bounded for $\int_{S}\left(\log ^{+}\left|g_{r}\right|\right) d \sigma$. While, when $r_{1}<r<1$, as $r \eta$ $\in D_{\alpha}(\eta)$, therefore $\left|g_{r}(\eta)\right| \leq\left(M_{\alpha} g\right)(\eta)$. Similar to " $\alpha \geq 2 ", \int_{S}\left(\log ^{+}\right.$ $\left.\left|g_{r}\right|\right) d \sigma$ is bounded. Summing up, for $\alpha>1$, there exists the implication $(b) \Rightarrow(a)$. 


\section{\$4. The John-Nirenberg Theorem in the Sense of Harmonic Measure}

For given $f \in L^{1}(\sigma)$, its Poisson extension to $B$ is also denoted by $f$, i. e.

$$
f(z)=\int_{S} P(z, \zeta) f(\zeta) d \sigma(\zeta)=\int_{S} f(\zeta) d \mu_{z}(\zeta) .
$$

In [7] we had defined a norm equivalent to the usual BMO norm

$$
\|f\|_{* *}=\sup _{a \in B} \int_{S}|f(\zeta)-f(a)| d \mu_{a}(\zeta) .
$$

If the Poisson extension of a BMO function is holomorphic in $B$, then we call this $f$ to be the holomorphic function with bounded mean oscillation (BMOA).

Theorem 3. $f \in B M O A \Leftrightarrow$ for every $a \in B$,

$$
\mu_{a}(\{\zeta \in S:|f(\zeta)-f(a)|>t\})<K e^{-\lambda_{1} t},
$$

where $K$ is an absolute constant, $\lambda_{1}=C /\|f\|_{* *}$.

Proof. " $\Rightarrow$ ". First of all, notice that $\left(M_{\alpha} g\right)(\eta) \geqq|g(\eta)|$, a. e. on $S$. In fact, by theorem 5.4. 8 and differentiation theorem (5.3.1) in [4], the $K$-limit of Poisson integral $f \circ \psi(z)$ of $L^{1}$ function $f \circ \psi(\eta)$ on $S$ is still $f \circ \phi(\eta)$. Thus $g^{*}$ is the $K$-limit of $g$,

$$
\begin{aligned}
g^{*}(\eta) & \triangleq(K-\lim g)(\eta) \\
& =(K-\lim f \circ \psi)(\eta)-f \circ \psi(0) \\
& =f \circ \psi(\eta)-f \circ \psi(0)=g(\eta), \text { a. e. on } S .
\end{aligned}
$$

Hence $\left(M_{\alpha} g\right)(\eta) \geq\left|g^{*}(\eta)\right|=|g(\eta)|$, a. e. on $S$.

If $f \in \mathrm{BMOA}$, applying the corollary 3 in [7], then $\mathscr{M}(f)$ is bounded in $H^{1}$. And again, using theorem 1 , and combining $\left(M_{\alpha} g\right)$ $(\eta) \geq|g(\eta)|$, then

$$
\begin{aligned}
& \mu_{a}(\{\zeta \in S:|f(\zeta)-f(a)|>t\})=\mu_{0}(\{\eta \in S:|f \circ \psi(\eta)-f \circ \psi(0)|>t\}) \\
= & \mu_{0}(\{\eta \in S:|g(\eta)|>t\}) \leq \mu_{0}\left(\left\{\eta \in S:\left(M_{\alpha} g\right)(\eta)>t\right\}\right) \\
< & K e^{-\lambda t},
\end{aligned}
$$

where $\lambda=C / C(\alpha) \sigma_{1}(f), \sigma_{1}(f)=\sup \left\{\|g\|_{H^{1}}: g \in \mathscr{M}(f)\right\}=\|f\|_{* * \cdot} \quad$ Thus setting $\lambda_{1}=C\|f\|_{* *}^{-1} \leq \lambda$, “ $\Rightarrow$ " is proved. 
" $\Leftarrow "$. Set $\Lambda(t)=\mu_{a}(\{\zeta \in S:|f(\zeta)-f(a)|>t\})$, by means of Lemma $2(\mathrm{i})$, then

$$
\int_{S}|f(\zeta)-f(a)| d \mu_{a}(\zeta)=\int_{0}^{\infty} \Lambda(t) d t<\int_{0}^{\infty} K e^{-\lambda_{1} t} d t<\infty
$$

Thus

$$
\|f\|_{* *}=\sup _{a \in B} \int_{S}|f(\zeta)-f(a)| d \mu_{a}(\zeta)<\infty . \quad \text { Q. E. D. }
$$

Corollary. For holomorphic function $f$ in $B$, the following are equivalent :

(a) $f \in B M O A$.

(b) $\mathscr{M}(f)$ is bounded in $N$.

(c) $\mathscr{M}(f)$ is bounded in $H^{p}, 0<p<\infty$.

(d) There exists $\rho=\rho_{f}>0$, such that

$$
\sup _{g \in \mathscr{M}(f)} \sup _{0<r<1} \int_{S} e^{\rho\left|g_{r}\right|} d \sigma<\infty .
$$

Proof. Since $H^{p} \subset N$, it is clear for the implication (c) $\Rightarrow$ (b). Now we prove $(\mathrm{b}) \Rightarrow(\mathrm{c})$. Set $\Lambda(t)=\mu_{0}\left(\left\{\eta \in S:\left(M_{\alpha} g\right)(\eta)>t\right\}\right)$. By means of Theorem 2 and Lemma 2(i), then for every $g \in \mathscr{M}(f)$, there is

$$
\begin{aligned}
& \int_{S}\left[M_{\alpha} g\right]^{p} d \mu_{0}=p \int_{0}^{\infty} t^{p-1} \Lambda(t) d t \\
& <K p \int_{0}^{\infty} t^{p-1} e^{-\lambda t} d t=\frac{K \Gamma(p+1)}{\lambda^{p}}<\infty .
\end{aligned}
$$

Similar to the treatment of proving (b) $\Rightarrow$ (a) in Theorem 2, distinguishing the cases of $\alpha \geq 2$ and $1<\alpha<2$, we have both

$$
\sup _{0<r<1} \int_{S}\left|g_{r}\right|^{p} d \sigma<C_{2}<\infty,
$$

for every $g \in \mathscr{M}(f)$, i. e. $\mathscr{M}(f)$ is bounded in $H^{p}, 0<p<\infty$.

The above assertion holds for every $H^{p}$ or for some $H^{p}$. Especially, when $1 \leq p<\infty$, since we have already supposed that the Poisson extension to $B$ of an $L^{1}$ function $g$ on $S$ is also denoted by $g$. By theorem 3.3.4. (b) in [4], it follows

$$
\|g\|_{H^{p}}^{p} \leq\|g\|_{L^{p}}^{p}=\int_{S}|g(\eta)|^{p} d \sigma(\eta)
$$




$$
\leq \int_{S}\left[\left(M_{\alpha} g\right)(\eta)\right]^{p} d \sigma<\frac{K \Gamma(p+1)}{\lambda^{p}} \leq \frac{K \Gamma(p+1)}{C^{p}}\|f\|_{* *}^{p} .
$$

Since $f \in \operatorname{BMOA} \Leftrightarrow \mathscr{M}(f)$ is bounded in $H^{1}$ (see [7]), thus (a) $\Leftrightarrow$ (b) is the special case of (b) $\Leftrightarrow$ (c) when $p=1$.

(d) $\Rightarrow$ (a) is quite an obvious fact. Since $\rho\left|g_{r}\right|<e^{\rho\left|g_{r}\right|}$, thus

$$
\|f\|_{* *}=\sup _{g \in \mathscr{M}(f)} \sup _{0<r<1} \int_{S}\left|g_{r}\right| d \sigma<\rho^{-1} \sup _{g \in \mathscr{M}(f)} \sup _{0<r<1} \int_{S} e^{\rho\left|g_{r}\right|} d \sigma<\infty .
$$

Now we would like to prove (a) $\Rightarrow(\mathrm{d})$. Suppose $\Lambda(t)=\mu_{a}(\{\zeta \in$ $S:|f(\zeta)-f(a)|>t\})$. Employing Theorem 3 and Lemma 3, then for every $a \in B$

$$
\begin{aligned}
& \int_{S} e^{\rho|g(\eta)|} d \sigma(\eta)=\int_{S} e^{\rho|f \circ \psi(\eta)-f \circ \psi(0)|} d \mu_{0}(\eta) \\
& =\int_{S} e^{\rho|f(\zeta)-f(a)|} d \mu_{a}(\zeta) \leq 1+\rho \int_{0}^{\infty} e^{\rho t} \Lambda(t) d t \\
& <1+K \rho \int_{0}^{\infty} e^{\left(\rho-\lambda_{1}\right) t} d t,
\end{aligned}
$$

it is only necessary to take $\rho<\lambda_{1}=C /\|f\|_{* *}$, then this integral converges.

On the other hand, when $0<r<1$,

$$
\begin{aligned}
& \int_{S} e^{\rho\left|g_{r}(\eta)\right|} d \sigma(\eta)=\int_{S}\left(e^{\rho\left|\int_{S} P(\eta \eta . \zeta) g(\xi) d \sigma(\xi)\right|}\right) d \sigma(\eta) \\
\leq & \int_{S}\left(e^{\rho \int_{S}|g(\xi)| d \mu_{r \eta}(\zeta)}\right) d \sigma(\eta),
\end{aligned}
$$

as the measure $\mu_{a}(S)=1$, so by Jensen's convexity inequality and noting $\int_{S} P(r \eta, \zeta) d \sigma(\eta)=1$ (see[4]), then for $0<r<1$

$$
\begin{aligned}
& \int_{S} e^{\rho\left|g_{r}(\eta)\right|} d \sigma(\eta) \\
\leq & \int_{S}\left(e^{\rho \int_{S}|g(\zeta)| d \mu_{r \eta}(\zeta)}\right) d \sigma(\eta) \leq \int_{S}\left(\int_{S} e^{\rho|g(\zeta)|} d \mu_{r \eta}(\zeta)\right) d \sigma(\eta) \\
= & \int_{S} e^{\rho|g(\zeta)|} d \sigma(\zeta) \int_{S} P(r \eta, \zeta) d \sigma(\eta) \\
= & \int_{S} e^{\rho|g(\zeta)|} d \sigma(\zeta) .
\end{aligned}
$$

Combining (11) and taking $\rho=\rho_{f} \in\left(0, C /\|f\|_{* *}\right)$, then for every $g \in \mathscr{M}(f)$

$$
\sup _{0<r<1} \int_{S} e^{\rho\left|g_{r}\right|} d \sigma<1+K \rho \int_{0}^{\infty} e^{\left(\rho-C\|f\|_{* *}^{-1}\right) t} d t<\infty
$$


(a) $\Rightarrow(d)$ is proved.

\section{References}

[1] John, F. \& Nirenberg, L., Comm. Pure Appl. Math., 14 (1961), 415-426.

[2] Baernstein, A., Aspects of Contemporary Complex Analysis, Academic Press, London and New York, 1980, 3-36.

[3] Long Ruilin \& Yang Le, Scientia Sinica, A27 (1984), 695-708.

[4] Rudin, W., Function Theory in the Unit Ball of $\boldsymbol{C}^{n}$, Springer-Verlag, New York, 1980.

[5] Korányi, A., Trans. Amer. Math. Soc., 135 (1969), 507-516.

[6] Ouyang Caiheng, Kexue Tongbao, 32 (1987), 9, 581-584.

[7] Acta Math. Sci., (Chin. Ed.), 7 (1987), 1, 103-110.

[8] Stein, E., Boundary Behavior of Holomorphic Functions of Several Complex Variables, Princeton Univ. Press, Princeton, NJ, 1972. 
